\title{
BRYOFLORISTICAL DATA FROM THE GUTÂI MOUNTAINS (ROMANIAN EASTERN CARPATHIAN, TRANSYLVANIA)
}

\author{
Andrea Sass-Gyarmati \\ Eszterházy Károly University, Institute of Biology, Department of \\ Botany and Plant Physiology; Eger, Pf. 43, H-3301 Hungary; \\ E-mail: sassgyarmati@gmail.com

\begin{abstract}
The main aim of this study was to explore the bryophyte diversity and distribution patterns in the Gutâi Mountains. From our collections hitherto 52 bryophyte species were identified. The 12 Marchantiophyta and 40 Bryophyta species belong to 45 genera of 27 families. Nardia scalaris is new for the whole Gutâi Mountains. Among them the vulnerable Grimmia muehlenbeckii and the very rare Riccardia palmata are worth to be mentioned.
\end{abstract}

Keywords: bryoflora, rare species, Gutâi Mountains, Romania

Rezumat: Lucrarea prezintă distribuţia speciilor de briofite din arealul Munţilor Gutâi. Din colecţia recentă au fost identificate 52 specii de briofite. Cele 12 specii de Marchantiophyta şi 40 de specii de Bryophyta aparţin la 45 de genuri şi 27 de familii. Nardia scalaris este o semnalare nouă pentru Munţii Gutâi. Grimmia muehlenbeckii este o specie vulnerabilă, iar Riccardia palmata este rară, ambele meritând a fi menţionate.

Cuvinte cheie: brioflora, specii rare, munţii Gutâi, Gutin, România

\section{INTRODUCTION}

First bryological records of the Gutâi (Gutin) Mountains were published at the end of XIXth century (Juratzka 1882) and the investigations continues in the $\mathrm{XX}^{\text {th }}$ century, which till now is far from complete (Pop 1942; Boros 1943, 1951; Boros and Vajda 1967; Raţiu and Moldovan 1972a, 1972b, 1974; Ştefureac 1974, 1976-1977; Mititelu and Dorca 1983; Coldea and Plămadă 1989). A very detailed floristical and vegetational study is given by Moldovan (1970) and one study was published on saxicolous 
lichens from the Gutâi Mountains (Codoreanu 1972). Tamás Pócs with his wife visited and collected in the area during the summer of 1993.

Much less bryological investigations has been done in the past twenty years completing with additional floristical data (Jakab 1999, Ardelean et al. 2008). We started our work in 2018 and our aim is to continue bryological exploration of this area.

\section{Study area}

The Gutâi Mountains are a mountain range within the Vihorlat Gutâi area of the inner Eastern Carpathians. Igniş and Gutâi mountains are situated at the western and southern limit of Maramureş Land, they are the oldest sector of the volcanic range in Eastern Carpathians. Separated by mountain passes from the neighbouring units (Huta 587 m, Gutâi 984 m, Neteda 1039 m) they are two separate units distinguished by geoforms originating from different types of volcanic activity: Ignis mountains as andesitic plateau, mostly stratified, with small depressions, an end cliff and residual forms, named rocks (Piatra Săpânței, Piatra Goală, Piatra Rea etc); Gutâi Mountains with pyroxene andesite, mostly vertical columns with a controversial neck - Creasta Cocoşului and cone shaped summits (Figure 1). On the northern limit of the mountains, a piedmont range forms contact with the Maramuress lowland, often associated with the mountain range due to the position of the settlements around the massifs (Ilies et al. 2017). The Gutâi Mountains have several higher regions: Gutâiul Mare (1443 m), Creasta Cocoșului (1395 m), Trei Apostoli (1398 m), Gutâiul Doamnei $(1426 \mathrm{~m})$ and Secătura $(1390 \mathrm{~m})$. Creasta Cocoșului is a protected area of national interest and is included in the GutâiCreasta Cocossului Natura-2000 site it is a ridge formation about 200 metres in length and located at an average altitude of 1200 metres, surrounded by mixed forests, large beechwood and spruce areas, the peat bog at Tăul Chendroaiei (Chendroaia's Pond), juniper areas and mountain pastures. The climate of the SE Carpathians is colder and more continental than that of the NW Carpathians (Hajdú-Moharos 1996). The Firiza Lake was established in 1964, when $52 \mathrm{~m}$ high dam gates were closed to stem the Firiza water tributary of the Sasar at Baia Mare. The lake has a length of $3 \mathrm{~km}$ and a width of $1 \mathrm{~km}$. Built for the Baia Mare city water supply, now the Firiza Lake is used for recreational and 
leisure and is one of the favorite places of population in Baia Mare, the landscape is particularly special, with coniferous and deciduous forest around.

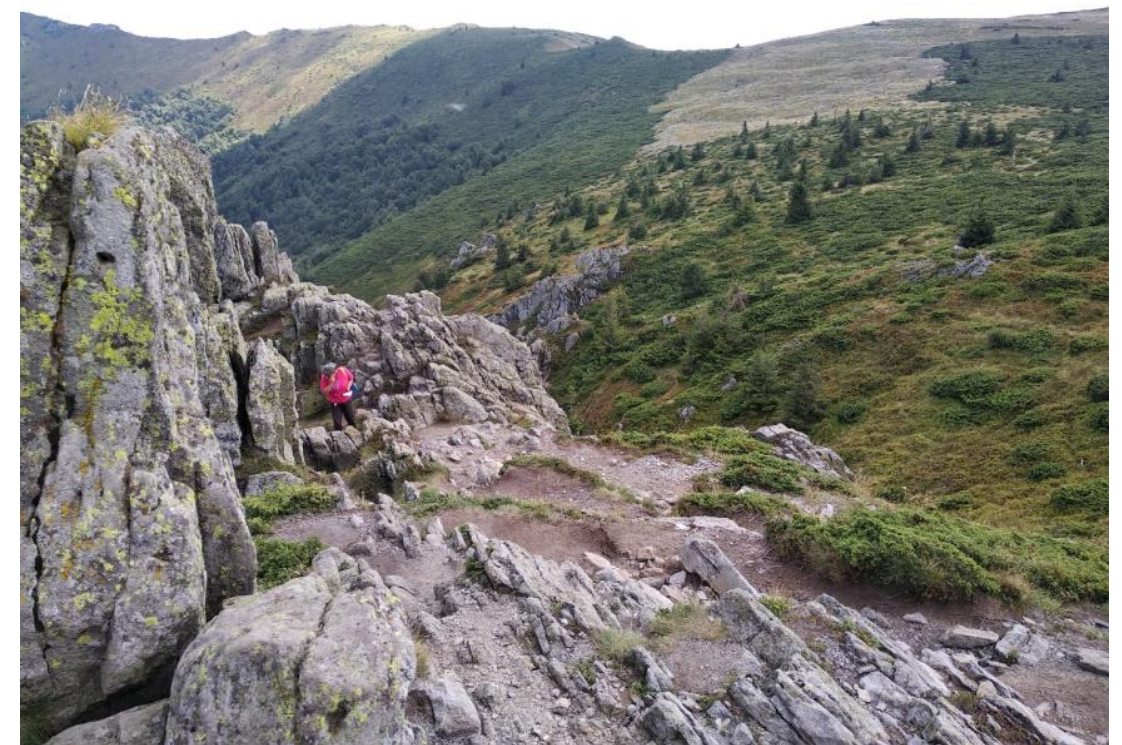

Figure 1. View from the Creasta Cocoșului summit (Photo: Róbert Sass-Gyarmati).

\section{MATERIAL AND METHODS}

The byophytes enumerated below were collected from the Gutâi Mountains between 8-9 August 2018 by Andrea and Róbert SassGyarmati and identified by Andrea Sass-Gyarmati and the species Grimmia muehlenbeckii identified by Peter Erzberger. The collection was made in various vegetation types: meadows, beech and spruce forests and subalpine belts. The Romanian distribution of mosses was established from Plămadă (1998) and Mohan (1998), while that of the liverworts from Ştefănuţ (2008). The nomenclature of liverworts follows Ştefănuţ (2008) modified by Söderström et al. (2016), nomenclature of mosses follows Hill et al. (2006), except Racomitrium affine which was recently included to Bucklandiella (F. Weber \& D. Mohr) Bednarek-Ochyra \& Ochyra (Ochyra et al. 2003). and Racomitrium aquaticum also recently included to Codriophorus (Brid. ex Schrad.) Bedn.-Ochyra \& Ochyra, 
Bednarek-Ochyra (2006). The classification of liverworts (Marchantiophyta) follows Söderström et al. (2016), while the classification of mosses (Bryophyta) follows Goffinet and Shaw (2009). The species in each family are arranged in alphabetical order. Species names are followed by the collecting site number, and by the substrate on which they were grown. The collected specimens are deposited in the Herbarium of Eger (EGR). The collecting sites are listed in the Appendix.

\section{RESULTS}

\section{List of species}

During the field study 52 bryophyte species were found in the investigated area. The 12 Marchantiophyta and 40 Bryophyta species belong to 45 genera of 27 families.

\section{Marchantiophyta}

Conocephalaceae

Conocephalum conicum (L.) Dumort. - 4: on irrigated rocks

Marchantiaceae

Marchantia polymorpha L. - 4: on irrigated rocks

Aneuraceae

Riccardia palmata (Hedw.) Carruth - 4: on decaying wood

Lophoziaceae

Lophozia ventricosa (Dicks.) Dum. - on decaying wood

\section{Scapaniaceae}

Diplophyllum albicans (L.) Dumort. - 5: on soil covered rocks Scapania undulata (L.) Dumort. - 4: on irrigated volcanic rocks

\section{Gymnomitriaceae}

Nardia scalaris Gray - 5: on soil. It was collected also by S. \& T. Pócs in 1993 (unpublished).

Marsupella emarginata (Ehrh.) Dumort. - 5: on soil

Radulaceae

Radula complanata (L.) Dumort. - 1, 4: bark of Fagus 


\title{
Lophocoleaceae
}

Chiloscyphus polyanthos (L.) Corda - 4: on irrigated volcanic rocks

Lophocolea heterophylla (Schrad.) Dumort. - 1: on decaying wood

Plagiochilaceae

Plagiochila porelloides (Torrey. ex Nees) Lindenb. - 4: on soil

\author{
Bryophyta \\ Andreaceae
}

Andreaea rupestris Hedw. - 6: on volcanic rocks

Tetraphidaceae

Tetraphis pellucida Hedw. - 6: on decaying wood

Polytrichaceae

Atrichum undulatum (Hedw.) P. Beauv. - 1, 4: on soil

Oligotrichum hercynicum (Hedw.) Lam. \& DC. - 3: on soil

Pogonatum urnigerum (Hedw.) P. Beauv. - 3: on soil

Polytrichastrum alpinum (Hedw.) G. L. Sm. - 5: on soil

Polytrichastrum formosum (Hedw.) G. L. Sm. - 2, 4: on soil

Polytrichum juniperinum Hedw. - 6: on rocks

Encalyptaceae

Encalypta streptocarpa Hedw. - 5: on soil

\section{Grimmiaceae}

Grimmia muehlenbeckii Schimp. - 6: on rocks

Codriophorus aquaticus (Brid.) Bednarek-Ochyra \& Ochyra. Syn.: Racomitrium aquaticum (Hedw.) Brid. - 4: on rocks

Bucklandiella affinis (F. Weber \& D. Mohr) Bednarek-Ochyra \& Ochyra. Syn.: Racomitrium affine (F. Weber et D. Mohr) Lindb. 6: on soil

\section{Ditrichaceae}

Ceratodon purpureus (Hedw.) - 1: on disturbed soil

\section{Dicranaceae}

Dicranella heteromalla (Hedw.) Schimp. - 2, 5: on decaying wood Dicranoweisia crispula (Hedw.) Milde - 4, 6: on volcanic rocks 
Dicranum flagellare Hedw. - 4: base of Fagus

Dicranum scoparium Hedw. - 1: base of Carpinus

Paraleucobryum longifolium (Hedw.) Loeske - 4: on soil covered rocks, 6: on rocks

\section{Pottiaceae}

Bryoerythrophyllum recurvirostrum (Hedw.) P. C. Chen - 5: on soil covered rocks

Didymodon fallax (Hedw.) R. H. Zander - 5: on soil

Gymnostomum calcareum Nees \& Hornsch. - 6: on vertical cliff

Bryaceae

Bryum pseudotriquetrum (Hedw.) P. Gaertn. - 3: on irrigated rocks

$$
\text { Mniaceae }
$$

Plagiomnium undulatum (Hedw.) T. J. Kop. - 4: on soil covered rocks

Rhizomnium punctatum (Hedw.) T. J. Kop. - 2, 4: on soil

Leskea polycarpa Hedw. - 4: on bark

Pseudoleskeella nervosa (Brid.) Nyholm - 4: on bark

Amblystegiaceae

Amblystegium serpens (Hedw.) Schimp. - 2, 4: on tree base Amblystegium subtile (Hedw.) Schimp. - 2: on tree base Sanionia uncinata (Hedw.) Loeske - 4: on tree base

\section{Hylocomiaceae}

Pleurozium schreberi (Willd. ex Brid.) Mitt. - 13: on soil

\section{Pterigynandraceae}

Pterigynandrum filiforme Hedw. - 4: on Fagus bark

\section{Thuidiaceae}

Abietinella abietina (Hedw.) M. Fleisch. - 1: on soil

\section{Brachytheciaceae}

Brachythecium rutabulum (Hedw.) Schimp. - 1: on soil Brachythecium rivulare Schimp. - 2: on wet soil 
Brachythecium salebrosum (Hoffm. ex F. Weber et D. Mohr.)

Schimp. - 1,4: on soil

Plagiotheciaceae

Plagiothecium denticulatum (Hedw.) Schimp. - 1: on tree base

Plagiothecium laetum Schimp. - 4: on tree base

Hypnaceae

Ctenidium molluscum (Hedw.) Mitt. - 4, 5: on rocks

Hypnum cupressiforme Hedw. - 1: on rocks

Lembophyllaceae

Isothecium myosuroides Brid. - 1: on tree base

\section{DISCUSSION}

The results of this study contributes to the knowledge of the biodiversity in Gutâi Mountains. The main reason for relatively high biodiversity is the variety of habitat types that can be found in this area.

Nardia scalaris Gray - circumboreal, mountain taxon it is not known from the Gutâi Mountains. Based on Mohan checklist occurs in Maramureşului Mountains: Vl. Jâjla, Turcul and several localities from the romanian Carpathians: Iezer Păpuşa Mountains, Bihor Mountains, Bucegi Mountains, Retezat Mountains, Cibinului Mountains and Mlaştina turbăria Cristişor.

Riccardia palmata (Hedw.) Carruth. - circumboreal, mountain species, it is reported only from one locality from Gutâi Mountains: Cheile Tătaru at Mara (Boros and Vajda 1967). Other reports from surroundings are from Borşa, Secului Valley, Sighet, Poiana Şarampoiului Forest, Mara, Runc Valley, Puzdra Mountain, (Boros and Vajda, 1967); between Tocila Valley and Băiuţ (Jakab 1999), well distributed in the Romanian Carpathians (Mohan 1998).

Grimmia muehlenbeckii Schimp. - is treated as vulnerable (VU) in Romania (Ştefănuţ and Goia 2012), it is known just from few localities in the country: jud. Alba: Vl. Galbina, Mtele Găina; jud. Gorj: Mţii Parâng: pasul Surduc; jud. Harghita: Munţii Hargita; jud. Hunedoara: Deva; jud. Maramureş: Muntele Pietrosul Rodnei; jud. 
Suceava: Mtele Ceardac. (Mohan 1998). These findings should enhance the knowdledge of bryoflora, the results emphasizes the importance of further research in this highly valuable area.

Acknowledgement - The author thanks to the grant EFOP-3.6.1-16-2016-00001 "Complex improvement of research capacities and services at the Eszterházy Károly University" for supporting this article. Highly appreciated the identification of Grimmia muehlenbeckii by Peter Erzberger. The author would like to thank to reviewers, Tamás Pócs and Péter Szücs (Eger) for their useful comments to the manuscript. Thanks are due to Róbert Sass-Gyarmati for his companion and help in the fieldwork.

\section{REFERENCES}

Ardelean, A., Karacsonyi, C. \& Mohan, G. (2008). Studiul briofitelor din unele mlaștini din nord-vestul țării. Analele Gradinii Botanice Universitare Macea 2: 7-15.

BEDNAREK-OChyRA, H. (2006). A taxonomic monograph of the moss genus Codriophorus P. Beauv. (Grimmiaceae). Polish Academy of Sciences, Institute of Botany, Krakow, 276 pp.

Boros, Á. (1943). A Lápos és a Kapnik szurdokának flórája. Scripta Botanica Musei Transilvanici 2: 141-149.

Boros, Á. (1951). Bryologische Beiträge zur Kenntnis der Flora von Ungarn und der Karpaten. Acta Biologica Academiae Scientiarum Hungaricae 2(1-3): 369-409.

Boros, Á. \& VAJDA, L. (1967). Bryologische Beiträge zur kenntnis der Flora Transilvaniens. Revue Bryologique et Lichénologique 35(1-4): 216-253.

Codoreanu, V. (1972). Flora si vegetaţia lichenologică saxicolă de la Cheile Tătarului (Maramureş). Contribuţii Botanice, Cluj-Napoca, 123-132.

ColdEA, GH. \& PlĂMADĂ, E. (1989). Vegetaţia mlaștinilor oligotrofe din Carpaţii româneşti (Cl. Oxycocco-Sphagnetea Br.-Bl. et Tx. 1943). Contribuţii Botanice, Cluj-Napoca 29: 37-43.

Goffinet, B. \& Shaw, A.J. (eds.) (2009). Bryophyte biology. Cambridge University Press, Cambridge, 565 pp.

Hajdú-Moharos, J. (1996). Az Északkeleti-Kárpátok. [The SE Carpathians.]. In: Karátson D. (ed.): Pannon Enciklopédia-Magyarország Földje. (Kitekintéssel a Kárpát-medence egészére). Keretek 2000 Kiadó, Budapest, pp. 371-373.

Hill, M.O., Bell, N., Bruggeman-Nannenga, M.A., Brugués, M., Cano, M.J., Enroth, J., FlatberG, K.I., Frahm, J.-P., Gallego, M.T., Garilleti, R., Guerra, J., Hedenäs, L., Holyoak, D.T., Hyvönen, J., Ignatov, M.S., LaRa, F., Mazimpaka, V., Muñoz, J. \& SöDERSTRÖM, L. (2006). An annotated checklist of the mosses of Europe and Macaronesia. Journal of Bryology 28: 198-267. https://doi.org/10.1179/174328206x119998

Ilies, M., IliEs, G., HoteA, M. \& Wend, J.A. (2017). Geomorphic attributes involved in sustainable ecosystem management scenarios for the Igniş-Gutâi Mountains Romania. Journal of Environmental Biology 38: 1121-1127.

http://doi.org/10.22438/jeb/38/5(SI)/GM-32 
JAKAB, G. (1999). Contributions to the knowledge of the bryophyte flora of the SE Carpathians (Romania). Studia Botanica Hungarica 29: 49-57.

JURATZKA, J. (1882). Die Laubmoosflora von Oesterreich-Ungarn. Handschriftlicher Nachlass Jakob Juratzka's zusammengestellt von J. Breidler und J. B. Förster. $K$. k. zoologisch-botanischen Gesellschaft in Wien 385 pp.

Mititelu, D. \& DoRCA, M. (1983). Flora şi vegetaţia a două rezervaţii botanice din Maramureş: "Lacul Morărenilor" şi "Tăul de sub Gutîi”. Analele ştiinţifice ale Universităţii "Al. I. Cuza” din Iaşi 39(2): 27-28.

Mohan, G. (1998). Catalogul briofitelor din România. Acta Botanica Horti Bucurestiensis. Ed. Univ. Bucureşti, 432 pp.

Moldovan, I. (1970). Flora şi vegetaţia Muntelui Gutâi. Universitatea „Babeş-Bolyai”, Teza de doctorat, Cluj Napoca.

Ochyra, R., Zarnowiec J. \& Bednarek-Ochyra H. (2003). Census Catalogue of Polish Mosses. Polish Academy of Sciences, Krakow, 372 pp.

PlăMADĂ, E. (1998). Flora briologică a României, Clasa Musci. Vol. I. Fasc. I. Sphagnales - Andreaeales - Tetraphidales - Buxbaumiales - Schistostegales Polytrichales - Fissidentales - Archidiales - Seligeriales. Cluj-Napoca: Presa Universitară Clujană, 230 pp.

Pop, E. (1942). Contribuţii la istoria pădurilor din nordul Transilvaniei. Buletinul Grădinii Botanice Cluj 22(1-4): 101-177.

Raţiu, 0. \& Moldovan, I. (1972a). Consideraţii cenologice asupra vegetaţiei mlaștinei Izvoarele (platoul vulcanic Gutâi-Oaş). Contribuţii Botanice, Cluj 12: 149-159.

RaţıU, O. \& Moldovan I. (1972b). Vegetaţia cheilor Tătarului (munţii Gutîiului). Studia Univ Babeș-Bolyai, Seria Biol., Cluj (1): 3-9.

RaŢıU, O. \& Moldovan, I. (1974). Consideraţii cenologice asupra vegetaţiei muntelui Igniș). Contribuţii Botanice, Cluj 14: 85-94.

SöDerström, L., Hagborg, A., Von Konrat, M., Bartholomew-Began, S., Bell, D., Briscoe, L., Brown, E., Cargill, D.C., Costa, D.P., Crandall-Stotler, B.J., Cooper, E.D., Dauphin, G., Engel, J.J., Feldberg, K., Glenny, D., Gradstein, S.R., He, X., Heinrichs, J., Hentschel, J., Ilkiu-Borges, A.L., Katagiri, T., Konstantinova, N.A., Larraín, J., Long, D.G., Nebel, M., Pócs, T., Puche, F., Reiner-Drehwald, E., Renner, M.A.M., Sass-Gyarmati, A., SchäFer-Verwimp, A., Moragues, J.G.S., Stotler, R.E., SukKharaK, P., Thiers, B.M., Uribe, J., VáŇA, J., Villarreal, J.C., Wigginton, M., Zhang, L. \& Zhu, R.-L. (2016). World checklist of hornworts and liverworts. PhytoKeys 59: 1828. https://doi.org/10.3897/phytokeys.59.6261

ŞTEFĂnuT, S. (2008). The Hornwort and Liverwort Atlas of Romania. Edit. Ars Docendi - Universitatea din Bucureşti, București, 510 pp.

ŞTEFĂNUŢ, S. \& GoIA I. (2012). Checklist and Red List of Bryophytes of Romania. Nova Hedwigia 95(1-2): 59-104.

https://doi.org/10.1127/0029-5035/2012/0044

ŞTEFUREAC, T. (1974). Semnificaţia unor noi şi valoroase briofite si angiosperme în rezervaţiile naturale din județul Suceava şi din unele ţinuturi învecinate. Studii si Cercetări Biologice 26(3): 165-170.

ŞTEFUREAC, T. (1976-1977). Noi contribuţii la ecologia si corologia sfagnaceelor din România. Studii şi Comunicări, Bacău 97-112.

(submitted: 01.10.2019, accepted: 09.10.2019) 


\section{APPENDIX}

List of collecting sites from the Gutâi Mts:

1. Munţii Gutâi (Gutin hegység), Maramureş County. Firiza Lake above Firiza village in acidophyllous Fagus-Carpinus forest at 5-600 m alt. $47^{\circ} 43^{\prime} 30.95^{\prime \prime} \mathrm{N}$, 2335'54.45”E. Date: 08. Aug. 2018. Coll.: A. \& R. Sass-Gyarmati No. 1801

2. Munţii Gutâi (Gutin hegység), Maramureş County. Gutin Pas (Pasul Gutâi). Acidophyllous beech forest (Luzulo-Fagetum) at $980 \mathrm{~m}$ alt. N47\%42'0.02", E2347'33.77". Date: 09. Aug. 2018. Coll.: A. \& R. Sass-Gyarmati No. 1802

3. Munţii Gutâi (Gutin hegység), Maramureș County. Spring bogs Poiana Boului (Ökörmező), NE from Baia Sprie (Felsőbánya), at $1055 \mathrm{~m}$ alt. N4741'49.37", E2348'13.03". Date: 09. Aug. 2018. Coll.: A. \& R. Sass-Gyarmati No. 1803

4. Munţii Gutâi (Gutin hegység), Maramureș County. Subalpine beech forest below the forest line along the path to Creasta Cocoşului Peak summit between 11001200 m alt. N 47²4'14.42", E 2350'28.66". Date: 09. Aug. 2018. Coll.: A. \& R. Sass-Gyarmati No. 1804

5. Munţii Gutâi (Gutin hegység), Maramureș County. Subalpine Vaccinium dwarf bush on the Creasta Cocoşului (Kakastaréj) summit at 1400-1420 m alt. N47²'14.22", E 2350'30.55". Date: 09. Aug. 2018. Coll.: A. \& R. SassGyarmati No. 1805

6. Munţii Gutâi (Gutin hegység), Maramureş County. Volcanic rocks above forest line near Creasta Cocoşului (Kakastaréj) crest at 1400 m alt. N47²4'14.55", E2350'30.53" Date: 09. Aug. 2018. Coll.: A. \& R. Sass-Gyarmati No. 1806 